\title{
Relación entre el funcionamiento familiar y el cumplimiento del cuidador en la atención a personas con autismo
}

\author{
Palomino-Moore Karla ${ }^{a}$, Vargas-Leo Mayra ${ }^{1, a}$, Vaiz-Bonifaz Rosa ${ }^{2, b, c}$
}

\section{RESUMEN}

Objetivo: determinar la relación entre el funcionamiento familiar y el cumplimiento del cuidador en la atención a personas con autismo. Material y métodos: estudio analítico, descriptivo y de corte transversal. La muestra fue de 31 cuidadores de personas con autismo. Para la recolección de los datos se usó como instrumentos, cuestionarios para determinar el funcionamiento familiar y el cumplimiento de la atención, previamente a la recolección de datos se sometió el instrumento utilizado a una prueba de confiabilidad a través de la prueba piloto. Para el procesamiento de datos se utilizó el programa SPSS y para el análisis estadístico la prueba de correlación de Pearson. Resultados: el desempeño familiar funcional en el hogar de la persona con autismo obtuvo un 51,6\% en el total de cuidadores, donde la dimensión «afectividad» alcanzó un mayor nivel (8,4 puntos). El 58,1\% presentó un cumplimiento eficiente en la atención a las personas con autismo y la dimensión cognitiva alcanzo un mayor nivel con 14,2 ptos., la correlación es $\mathrm{r}=0,445$. Conclusiones: la relación entre el funcionamiento familiar y el cumplimiento del cuidador en la atención a las personas con autismo presentan una correlación positiva y significativa, el cual indica que a puntajes altos de funcionamiento familiar le corresponde puntajes altos de cumplimiento en la atención y viceversa en puntajes bajos.

Palabras clave: trastorno autístico, relaciones familiares, atención. Perú (Fuente DeCs BIREME).

\section{Relationship between family functioning and compliance of the caregiver in the care of people with autism}

\begin{abstract}
Objetive: To determine the relationship between family functioning and compliance of the caregiver in caring for people with autism. Material and Methods: Analytical, descriptive and cross-sectional studio. The sample consisted of 31 caregivers of people with autism. For data collection questionnaire was used as tools to determine family functioning and compliance of care prior to the data collection instrument used to test reliability through the pilot was subjected. For data processing we used SPSS and statistical analysis was Pearson correlation test. Results: Showed that functional family functioning in the home of the person with autism scored one $(51.6 \%)$ of all careers. Being affectivity dimension who reached a higher level (8.4 points). The $(58.1 \%)$ presented efficient fulfillment in caring for people with autism and cognitive dimension reached a higher level (14.2ptos). Conclusions. The relationship between family functioning and compliance with the caregiver in caring for people with autism have a positive and statistically significant correlation, indicating that higher scores on family functioning corresponds compliance high scores on attention and vice versa in low scores.
\end{abstract}

Key words: Autism, family functioning, attention. Peru (Source DeCs BIREME).

\footnotetext{
${ }^{1}$ Clínica Salud Ocupacional Medical Assistant. Lima,Perú.

2. Facultad e Enfermería, Universidad Peruana Cayetano Heredia. Lima, Perú.
} 


\section{INTRODUCCIÓN}

El trastorno autista de acuerdo con el Manual Diagnóstico y Estadístico de los Trastornos Mentales (DSM-IVTR 2000), implica un inicio temprano de alteración en la interacción social, déficits de comunicación y un repertorio restringido de actividades e intereses. El autismo se define como un trastorno del desarrollo, resultado de un desorden neurológico, que afecta el funcionamiento del cerebro, compromete básicamente las áreas de interacción social, conductual y de lenguaje alterando el procesamiento de la información recibida mediante los sentidos, lo que produce una reacción ante algunas sensaciones, imágenes, sonidos y olores. Suele presentarse las manifestaciones clínicas de distintas maneras entre las edades de 15 meses a 3 años (1).

Al nacer el niño, los padres sienten que es uno de los acontecimientos vitales más importantes de su vida e idealizan un futuro para él. Sin embargo, en algunos niños a partir del primer año de vida, empiezan a aparecer ciertos comportamientos inusuales, los cuales preocupan a los padres, y cuando se les informa que su hijo sufre de autismo infantil, el impacto emocional es grande. Esto ocurre debido a la preocupación de los padres con respecto al crecimiento y desarrollo de su hijo, lo cual ocasiona cambios en el entorno familiar (2).

La familia es un grupo natural que en el transcurrir del tiempo elabora sus propias pautas de interacción dirigidas a consolidar los roles y funciones que le competen a cada uno de sus miembros (3). La unión de personas que comparten un proyecto vital de existencia común, en el que se generan fuertes sentimientos de pertenencia a dicho grupo, existe un compromiso personal entre sus miembros y se establecen intensas relaciones de intimidad, reciprocidad y dependencia» (4).

La convivencia en el hogar de un hijo con autismo suele ser difícil, las familias se ven sometidas desde el principio a modificaciones severas de su régimen de vida habitual debido a que el niño tiene limitaciones de su independencia, alteraciones del lenguaje, comportamiento agresivo, carencia de habilidades sociales, limitación de oportunidades de vida aunado a la falta de profesionales expertos para brindar el tratamiento y el consejo adecuado; todo ello influye en el sufrimiento e incomprensión de los padres, y en especial de la madre, así como un aumento en las responsabilidades, la pérdida de expectativas de control sobre la conducta del hijo, el miedo al futuro y la pérdida del trabajo (5). Los padres tienen que aprender a convivir con un hijo de características especiales que exi- ge adaptaciones importantes en el entorno familiar. Por ello, es preciso que comprendan el trastorno, que conozcan los síntomas y sus manifestaciones en la vida cotidiana. En cuanto a los sentimientos de los padres, deben tener en cuenta que estos son normales, que otros padres de hijos autistas han pasado por lo mismo y consiguieron sobreponerse; esa experiencia puede ayudar a otros padres a superar sus dificultades (6).

El funcionamiento familiar es el resultado de los esfuerzos de sus miembros para conseguir un nivel de equilibrio, armonía y coherencia ante una situación de crisis (7).No existe un funcionamiento familiar único o similar para cada familia, esto depende de la dinámica y comunicación. Martínez Bilbao refiere que el funcionamiento familiar se puede ver afectado por varios factores: la autoatribución de tareas o roles entre el padre y la madre en cuanto al cuidado del hijo con autismo y el grado de sensibilidad de cada uno de los miembros de la familia (2). Establecer roles para cada integrante, significa que cada uno cumpla con sus obligaciones, pero se hagan merecedores de sus derechos. Así, puede existir un alto grado de solidaridad y preocupación que los impulse a cooperar y ayudarse mutuamente (8).

La tendencia de la familia debe ser democrática, el liderazgo pertenece a uno o ambos padres, quienes se apoyan mutuamente en sus actitudes respecto a la crianza de sus hijos; la unión familiar se basa en afinidades y metas comunes no en el propósito de perjudicar a nadie. Las familias funcionales emplean sus recursos para tratar eficazmente los problemas del hijo con autismo y, al mismo tiempo, se muestran preocupados por las necesidades emocionales de cada miembro de la familia. Tener expectativas realistas con respecto al hijo con autismo y mantener un clima familiar de calidad son dos aspectos fundamentales en la convivencia positiva de la familia que previenen de posibles complicaciones a nivel personal, familiar y social. Los Indicadores del funcionamiento familiar son los siguientes: cohesión, armonía, comunicación, permeabilidad, afectividad, roles y adaptabilidad (9).

La cohesión es la unión familiar física y emocional al enfrentar diferentes situaciones, y la toma de decisiones de las tareas cotidianas. La armonía, corresponde a los intereses y necesidades individuales de la familia para un equilibrio emocional positivo. La comunicación es la capacidad de las familias de trasmitir sus experiencias de forma clara y directa. La permeabilidad es la capacidad de la familia de brindar y recibir experiencias de otros miembros e instituciones. La afectividad, se da cuando los miembros de la familia vivencian y demuestran sentimientos y 
emociones positivas unos a los otros. Los roles, se refiere a que cada miembro de la familia cumple las responsabilidades y funciones negociadas por el núcleo familiar. La adaptabilidad es la habilidad de la familia para cambiar de estructura de poder, relación de roles y reglas ante una situación que lo requiera (10).

Las personas con autismo requieren de un cuidado especial pues ellos necesitan aprender habilidades para su independencia, por eso es significativa la atención del cuidador. Otro aspecto importante de la atención a la persona con autismo es la necesidad de trabajar de la mano con los profesionales que les enseña y educa cada día, por su parte, el cuidador aprenderá ciertas actividades o tareas del día para aplicarlas en su hogar. La atención engloba actividades o acciones que se realizan para conseguir el resultado que uno desee, como el mejorar la calidad de vida y la integración social de las personas con autismo (11).

La atención del cuidador en la terapia tampoco debe ser excesiva, es más importante la calidad de atención que el tiempo. Si el cuidador se incorpora en la atención que brindan los terapistas, entonces entenderá la labor realizada e incrementará la colaboración en las atenciones en el hogar.

Los servicios de atención en el hogar están diseñados para satisfacer las necesidades de desarrollo de cada persona con autismo y su familia, y tienen como propósito aumentar el desarrollo de las personas con autismo y minimizar su retraso, así como aumentar la capacidad de las familias para satisfacer las necesidades especiales de las personas con autismo, sin ningún impedimento (12). La intervención de las educadoras y terapistas en su primer encuentro con el cuidador es ayudar a la integración de la persona con autismo en la familia. Dentro de las tareas que debe realizar el cuidador en el hogar están: la atención social, cognitiva y psicomotriz. La atención social es la acción conjunta, iniciativa de interacciones y de estados emocionales ante actividades con otros. La persona con autismo requiere de estas acciones para su aprendizaje, pues si no las tiene dominadas, el avance en las otras áreas será lento o casi nulo. A medida que el niño va interactuando en los juegos y con los juguetes, se va favoreciendo el desarrollo de la comunicación y el intercambio, preparando al niño para relacionarse con otros (13).

La atención cognitiva es el aprendizaje y adquisición de hábitos básicos para su independencia. Estos aprendizajes se van adquiriendo y consolidando poco a poco en situaciones de socialización; es decir, realizándolos con los demás. Su adquisición proporciona a las personas con autismo sentimientos de seguridad, de control de sí mismos y del medio, de identidad personal y el desarrollo de normas. Las actividades que enseñan a las personas con autismo son guardar su lonchera, cambiarse de ropa, lavarse las manos, etc. Estas tareas ayudan a que las personas autistas se desenvuelvan mejor en las actividades de su hogar (14).

La atención psicomotriz es el conjunto de herramientas corporales que incluyen el tono muscular, el ritmo, el equilibrio, el movimiento, la respiración y el gesto, destinados a incrementar el conocimiento del cuerpo en el espacio que le rodea. Se considera que la actividad motriz establece un nexo entre el pensamiento y la acción, y que un buen desarrollo psicomotor facilita la expresión y comunicación del lenguaje corporal, mejora el conocimiento y la identidad personal y favorece la relación con el espacio (15). Para esto, es importante que el cuidador participe en talleres de sensibilización, donde se les alienta a que desarrollen mecanismos positivos de afrontamiento, a que se sientan bien con ellos mismos y logren ser conscientes de la realidad de sus hijos (16). El objetivo de este trabajo fue determinar la relación entre el funcionamiento familiar y el cumplimiento del cuidador en la atención a personas con autismo.

\section{MATERIAL Y MÉTODOS}

Estudio analítico, de tipo cuantitativo y de corte transversal. Se desarrolló en el C.E.E. Pedro José Triest, en donde asisten 70 personas con problemas de trastorno neurológico (autismo, síndrome de Down, retraso mental, etc.), perteneciente a la jurisdicción de Lima-Norte en el distrito de Carabayllo. Se consideraron 31 cuidadores de las personas con autismo. Los criterios de selección fueron edad del cuidador mayor de 18 años que acepte participar en el estudio de investigación, firmando el consentimiento informado. Los datos se recolectaron con los siguientes cuestionarios:

a). Test de funcionamiento familiar (FF-SIL) (17), el cual fue adaptado para cuidadores de personas con autismo, el cuestionario evaluó las dimensiones: cohesión, armonía, comunicación, permeabilidad, afectividad, roles y adaptabilidad.

b). Cuestionario de cumplimiento del cuidador en la atención: fue construido y elaborado por las autoras del proyecto, se contó con la evaluación de una psicóloga, enfermera de entrenamiento grupal de personas con autismo del Instituto de Salud Mental 
Honorio Delgado e Hideyo Noguchi así como el director del colegio. El cuestionario de 15 ítems fue para evaluar el cumplimiento del cuidador en la atención de las personas con autismo, el cual evaluó la atención social, cognitiva y psicomotriz.

Los datos fueron codificados e ingresados a una base de datos creada en el programa estadístico SPSS versión 15 (programa estadístico para las ciencias sociales) para su análisis. Para determinar la relación entre el funcionamiento familiar y el cumplimiento del cuidador en la atención a las personas con autismo, se evaluó la correlación entre dichas variables por medio del coeficiente de correlación de Pearson. Estos, a su vez, fueron organizados en tablas de distribución de frecuencia y gráficos según cada objetivo, con la finalidad de visualizar y explicar los resultados de manera clara y precisa.

El proyecto fue aprobado por el Comité de Ética de la Universidad Peruana Cayetano Heredia, cada participante firmó el consentimiento informado, se consideró la confidencialidad de los datos y los principios éticos.

\section{RESULTADOS}

Tabla 1. Datos sociodemográficos del cuidador, febrero de 2013

\begin{tabular}{lrc}
\hline Datos socio demográficos & Número & $\begin{array}{c}\text { Porcentaje } \\
(\%)\end{array}$ \\
\hline Edad & & \\
$\quad$ Menor de 30 años & 6 & 19,4 \\
De 31 a 50 años & 21 & 67,7 \\
$\quad$ Mayor a 51 años & 4 & 12,9 \\
Sexo & & \\
$\quad$ Femenino & 27 & 87,1 \\
$\quad$ Masculino & 4 & 12,9 \\
Estado civil & & \\
Casado & 16 & 51,6 \\
Conviviente & 10 & 32,3 \\
$\quad$ Soltero & 5 & 16,1 \\
Grado de instrucción & & \\
$\quad$ Superior técnica & 18 & 58,5 \\
$\quad$ Secundaria & 13 & 41,5 \\
\hline
\end{tabular}

En los resultados se evidenció que la moda de las edades de los 31 cuidadores es de 36 años, el grupo etario se encuentra dentro de las edades de 31 a 50 años con $67,7 \%$. Prevalece el sexo femenino (87,1\%); el estado civil más frecuente es el casado $(51,6 \%)$, en menor cantidad padres solteros; el grado de instrucción alcanzado por el grupo fue superior técnica con $58,9 \%$.
Gráfico 1. Funcionamiento familiar en familias con personas con autismo, febrero de 2013

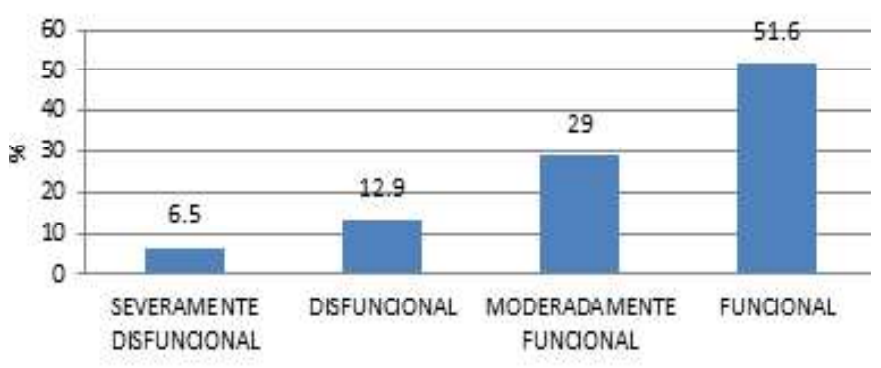

hogar de la persona con autismo obtuvo un $51,6 \%$ del total de cuidadores entrevistados.

Tabla 2. Funcionamiento familiar en familias con personas con autismo según dimensiones, febrero de 2013

\begin{tabular}{lcc}
\hline \multicolumn{1}{c}{ Dimensión } & $\overline{\mathrm{x}}$ & $\mathbf{s}$ \\
\hline Afectividad & 8,4 & 1,7 \\
Armonía & 7,9 & 2,2 \\
Permeabilidad & 7,4 & 2,1 \\
Comunicación & 7,3 & 2,4 \\
Cohesión & 7,3 & 2,3 \\
Roles & 7,3 & 2,3 \\
Adaptabilidad & 7,3 & 2,6 \\
\hline
\end{tabular}

El funcionamiento familiar consta de dimensiones, como se ilustra en la tabla 2, la dimensión afectividad fue la que alcanzó un mayor nivel de funcionalidad con un puntaje promedio de 8,4 puntos, sobre un máximo de 10 puntos $\mathrm{y}$ el resto de dimensiones se encuentra entre una variación de 7,9 a 7,3 puntos.

Gráfico 2. Cumplimiento de la atención a las personas con autismo, febrero de 2013

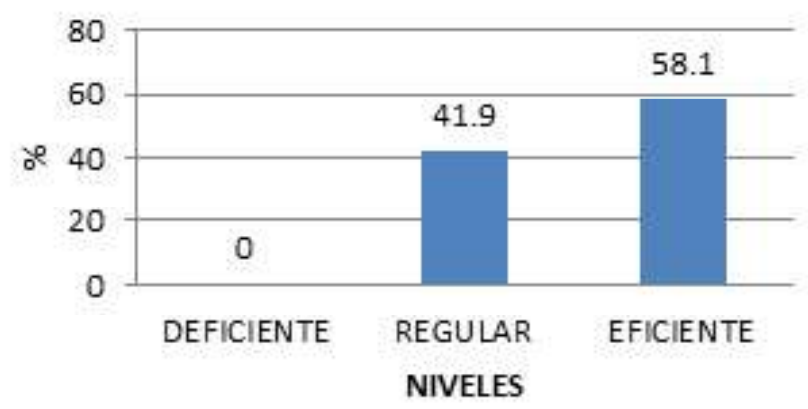

El 58,1\% de las familias presentó cumplimiento eficiente en la atención a las personas con autismo, mientras que en el $41,9 \%$ restante el cumplimiento fue regular. No se evidenció deficiencia en el cumplimiento de la atención de los cuidadores. 
Gráfico 3. Puntaje de promedios del cumplimiento de la atención a las personas con autismo en el hogar según dimensiones, febrero de 2013

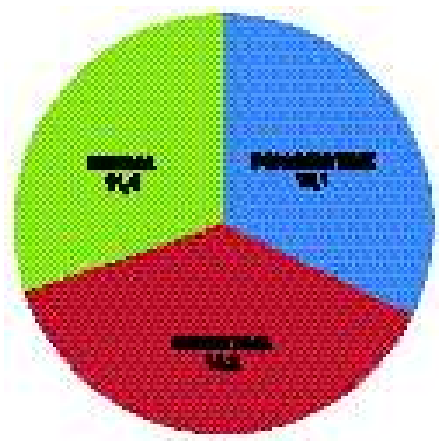

El cumplimiento de la atención consta de dimensiones, como se ilustra en el gráfico 3, la dimensión cognitiva alcanzó un mayor nivel de cumplimiento con un puntaje promedio de 14,2 pts., seguida de psicomotriz con 12,1 pts. La dimensión social cumplió un menor nivel con un puntaje promedio de $11,4 \mathrm{pts}$

\section{Gráfico 4. Relación entre el funcionamiento familiar y} cumplimiento de la atención, febrero de 2013

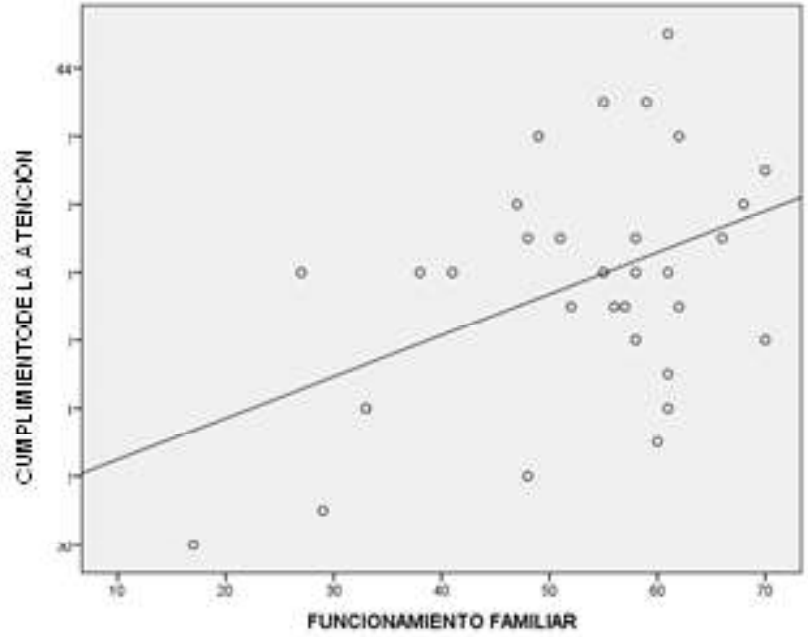

El coeficiente de correlación de Pearson $(r=0,445)$ evidenció una correlación positiva entre el funcionamiento familiar y el cumplimiento del cuidador en la atención a personas con autismo, la cual resultó estadísticamente significativa $(p=0,012)$, lo que significa que a puntajes altos de funcionamiento familiar le corresponden puntajes altos de cumplimiento en la atención del cuidador y a puntajes bajos de funcionamiento familiar le corresponden puntajes bajos de cumplimiento en la atención del cuidador.

\section{DISCUSIÓN}

En cuanto a las características sociodemográficas del cuidador de las personas con autismo, se evidenció mediante la moda que las edades de los 31 cuidadores son 36 años, prevaleció el grupo etario de 31 a 50 años, el sexo femenino, estado civil casado y grado de instrucción superior técnica.

En cuanto a las características sociodemográficas del cuidador de las personas con autismo, el $67,7 \%$ es de 31 a 50 años, este resultado está relacionado a que el promedio de edad de los cuidadores fue de 36 años según la moda, la menor fue 24 años mientras que la mayor fue 60 años. El 87,1 \% es de sexo femenino; el 51,6 \% de cuidadores son casados, este resultado nos indica que los padres se encuentran juntos para el cuidado de su hijo con autismo; el 58,9 \% tiene grado de instrucción superior técnica, lo cual atribuye que hay mayor facilidad de comprensión y conocimiento acerca del autismo.

Cuando se habla de funcionamiento familiar, se refiere a una dinámica relacional sistémica que se establece entre los miembros de una familia permitiendo que esta funcione positivamente, cumpla sus funciones y permita la expresión y desarrollo de la individualidad. Al observar en los resultados un mayor porcentaje de familias funcionales nos permite evidenciar el liderazgo de las familias que pertenece a uno o ambos padres quienes se apoyan mutuamente en sus actitudes respecto a la crianza de sus hijos, la unión familiar y metas comunes. A estos resultados atribuimos que el entorno de una familia de una persona con autismo puede incrementar los problemas y crear cambios importantes en el funcionamiento familiar (15). Ambas dimensiones son importantes para un funcionamiento familiar funcional, debido a los ítems de esa dimensión, la familia vivencia, demuestra sentimientos y emociones positivas unos a los otros. La unión de la familia para satisfacer los intereses y necesidades individuales conllevan a un entorno feliz (16).

El cumplimiento eficiente en la atención a personas con autismo se atribuye al esfuerzo y participación activa de los cuidadores en las charlas y visitas domiciliarias que realiza el C.E.E. Pedro Triest. Aprender a comprender el mundo a partir de la mente de su hijo, influye no solo en los cuidadores sino en toda la familia. Esto permite superar los problemas que impiden que las relaciones entre la familia y personas con autismo sean gratificantes y las atenciones efectivas. La atención continua en las terapias y en casa son factores determinantes para que la persona con autismo mejore la evolución de sus áreas afectadas, y por ende la independencia de sus actividades, así también, se inculca la realización de actividades formativas para lograr respuestas individuales en la forma de pensar, sentir y actuar, para favorecer las relaciones con el ambiente y el crecimiento de las potencialidades (18). 
El 41,9\% de las familias presentan un cumplimiento regular, los comportamientos de la persona con autismo tiene impacto negativo en el equilibrio familiar, principalmente porque los cuidadores no se encuentran emocionalmente preparados en la mayoría de los casos, experimentando múltiples y variadas reacciones que van desde tristeza hasta desesperación por no saber cómo actuar frente a la situación. Los cuidadores requieren de apoyo profesional, por lo cual recurren a centros o instituciones especializadas para obtener información que les permita aprender a lidiar con las personas con autismo y mejorar las áreas afectadas, fortaleciendo la independencia de la persona con autismo para poder integrarlo en la vida familiar y social. Los servicios de atención temprana están centralizados en la familia, es decir, están diseñados para satisfacer las necesidades de desarrollo de cada estudiante elegible y las necesidades de su familia, relacionadas con el fortalecimiento del desarrollo del autista.

El C.E.E. Pedro Triest realiza visitas domiciliarias y charlas a los cuidadores lo cual refuerza los cuidados que se debe tener e informan los avances y desarrollo de cada autista. En relación a las dimensiones del cuestionario del cumplimiento en la atención, la dimensión cognitiva fue la que alcanzó un mayor nivel de cumplimiento con un puntaje promedio de 14,2 puntos, seguida de la psicomotricidad con 12,1 puntos mientras que la dimensión social fue la que se cumplió en menor nivel con un puntaje promedio de 11,4 puntos (gráfico 3).

La dimensión cognitiva presentó un mayor nivel de cumplimiento con un puntaje promedio de 14,2 puntos, los cuidadores brindan apoyo a las personas con autismo para que sean más independientes, enseñándoles su autocuidado y que se desenvuelvan positivamente en sus actividades del hogar. Las educadoras del C.E.E. Pedro Triest enseñan a las personas con autismo estas tareas específicas para su independencia, para despertar el interés y lograr la confianza en el autista.

La dimensión psicomotriz presentó un puntaje promedio de 12,1 puntos, esto se atribuye a que los cuidadores no realizan con mayor frecuencia acciones intencionadas a través del movimiento para promover el desarrollo y la integración de las diferentes funciones de la psicomotricidad de la persona autista.

La dimensión social presentó un menor nivel de cumplimiento, con un puntaje promedio de 11,4 puntos esto puede desencadenar conflictos interiores, atravesando diversas etapas de adaptación, desde la fase de negación y rechazo de la persona autista hasta la aceptación propia- mente dicha. Este tipo de juegos tiene un sentido espontáneo e imaginario para las personas autistas. Esto es un motivo importante para considerar que dentro de las actividades de juego hay aprendizaje.

Es importante que el cuidador participe en talleres de sensibilización, donde se les aliente a que desarrollen mecanismos positivos de afrontamiento, a que se sientan bien con ellos mismos y logren ser conscientes de la realidad.

El estudio de la relación entre el funcionamiento familiar y el cumplimiento del cuidador en la atención a personas con autismo evidenció una correlación positiva $(\mathrm{r}=0,445)$ y estadísticamente significativa $(\mathrm{p}=0,0012)$ el cual indica que a puntajes altos de funcionamiento familiar le corresponde puntajes altos de cumplimiento en la atención y viceversa en puntajes bajos (gráfico 4).

Se concluye que el funcionamiento familiar de los cuidadores de personas con autismo presenta un desempeño funcional.

\section{REFERENCIAS BIBLIOGRÁFICAS}

1. Cabrera D. Generalidades sobre el autismo. Revista Colombiana de Psiquiatría. 2007. Bogotá. [citado 15 Feb 2010]. Disponible en: http://www.scielo.org.co/ scielo.php?pid=S003474502007000500016\&script=sci_antext.

2. Martínez M, Bilbao L. Acercamiento a la realidad de las familias de personas con autismo. Colegio Oficial de Psicólogos de Madrid. 2008. España. [citado 20 ene 2010]. Disponible en: http://scielo.isciii.es/ scielo.php?script $=$ sci_arttext $\quad \&$ pid $=$ S113205592008000200009 \&lang $=$ pt.

3. Ramos MA. ¿Qué es el autismo?: La experiencia de padres inmersos en la incertidumbre. Intersecciones antropol. 2010:11(1): 73-88. Disponible en:http:// www.scielo.org.ar/pdf/iant/v11n1/v11n1a06.pdf.

4. Almeida Nogueira M, Moreira Martins do Rio SC.A família com criança autista: apoio de enfermagem.Revista Portuguesa de Enfermagem de Saúde Mental 2011; 5:16-21. Disponible en:http:// www.scielo.gpeari.mctes.pt/pdf/rpesm/n5/n5a03.pdf

5. Anchante G, Burgos M, Tarazona S, Ahumada E. Conocimiento sobre autismo y cuidados del niño en una asociación de padres de familia. Rev. Enferm. Herediana. 2008; 1(1): 4-8. [citado 15 Sep 2009]. Disponible en: http://www.upch.edu.pe/vrinve/dugic/ public/pix/pub_ar_cientifica_mod2.pdf

6. Rodríguez E. Asociación de Padres y Terapeutas. La escuela de padres como respuesta a las necesidades de las familias con hijos con autismo. 
2008. España. [citado 29 May 2010].Disponible en: http://www.aetapi.org/congresos/santander_02/ comun_01.pdf

7. AvilaBeltran F, Soliz H. Impacto psicosocial del autismo en la familia. GacMed Bol 2006; 29 (1):2127. Disponible en:http://www.scielo.org.bo/pdf/gmb/ v29n1/a05.pdf

8. Zaldívar Pérez D.Funcionamiento familiar saludable. Infomed. 2010. Disponible en: http://www.sld.cu/ galerias/pdf/sitios/rehabilitaciontemprana/ funcionamiento_familiar.pdf

9. Rodríguez Torrens E. Atención a familias en los servicios para personas con trastornos del espectro autista. Educación y futuro.2010; 14:109-130.

10. Henriques Marques M, Rodrigues Dixe M. Crianças e jovens autistas: impacto na dinâmica familiar e pessoal de seus país.RevPsiqClín. 2011; 38(2):66-70. Disponible en:http://www.hcnet.usp.br/ipq/revista/ vol38/n2/66.htm.

11. Hernandez R, Risquet A, Perez P. Propuesta para mejorar la atención a niños y adolescentes autistas. 2010; 299-301. Cuba. [citado 20 feb 2013]. Disponible en: http://medicentro.sld.cu/index.php/medicentro/ article/viewFile/159/188

12. Vargas J, González C. Escuela para padres: el funcionamiento familiar. Centro Regional de Investigación en Psicología. 2008; 19- 22; 19- 20. México. [citado 18 may 2010] .Disponible en: http:// www.conductitlan.net/44_funcionamien to_familiar.pdf

13. NDSS. Miami. ¿Qué es la intervención temprana? 2010 [citado 12 diciembre 2011]. Disponible en:http:/ /esp.ndss.org/index.php?option=com_con tent\&view=article\&id=254:ique-es-la-intervenciontemprana\&catid $=72$ :intervencion-temprana\& Itemid $=82$
14. Educación Especial en Puerto Rico. Puerto Rico. Intervención Temprana. 2004. [citado 20 dic 2011]. Disponible en:http://edicacionespecialpr.tripod.com/ id32.html

15. Bueno Hernández A, Cárdenas Gutiérrez M, Pastor Zamalloa M, Silva-Mathews Zulema. Experiencias de los padres ante el cuidado de su hijo autista.Revenferm Herediana. 2012; 5(1):2635.Disponible en: http://www.upch.edu.pe/faenf/ images/stories/articulorevista5/autismo4

16. Benites Morales L.Autismo, familia y calidad de vida.Cultura: Lima (Perú) 2010; 24: 1-20. Disponible en:http://www.fcctp.usmp.edu.pe/cultura/imagenes/ pdf/24_07.pdf.

17. Cuba M, Jurado A, Romero Z, Cuba M.Características familiares asociadas a la percepción de la calidad de vida en pobladores de un área urbanomarginal en el Distrito de Los Olivos, Lima.RevMedHered2013;24 (1):12-16. Disponible en:http://www.scielo.org.pe/pdf/rmh/v24n1/ v24n1ao2.pdf

18. Herrera P. Rol de género y funcionamiento familiar. Revista Cubana de Medicina General Integral. [Serie en Internet]. 2000 [Citado 16 May 2010]. 16(6):56873. Disponible en: http://www.bvs.sld.cu/revistas/mgi/ vol16_6_00/mgi08600.html

\section{Correspondencia}

Rosa Vaiz Bonifaz

Universidad Peruana Cayetano Heredia

Dirección: Av. Honorio Delgado 430, Urb. Ingeniería, Lima - 31

Correo electrónico: rosa.vaiz@upch.pe

Forma de citar este artículo: Palomino-Moore K, Vargas-Leo M, Vaiz-Bonifaz R. Relación entre el funcionamiento familiar y el cumplimiento del cuidador en la atención a personas con autismo. Rev enferm Herediana. 2014;7(2):56-62. 